\title{
Looking beyond COVID-19 vaccine phase 3 trials
}

\author{
Jerome H. Kim $\odot^{1 凶}$, Florian Marks $s^{1,2,3}$ and John D. Clemens $s^{1,4,5}$
}

\begin{abstract}
After the recent announcement of COVID-19 vaccine efficacy in clinical trials by several manufacturers for protection against severe disease, a comprehensive post-efficacy strategy for the next steps to ensure vaccination of the global population is now required. These considerations should include how to manufacture billions of doses of high-quality vaccines, support for vaccine purchase, coordination of supply, the equitable distribution of vaccines and the logistics of global vaccine delivery, all of which are a prelude to a massive vaccination campaign targeting people of all ages. Furthermore, additional scientific questions about the vaccines remain that should be answered to improve vaccine efficacy, including questions regarding the optimization of vaccination regimens, booster doses, the correlates of protection, vaccine effectiveness, safety and enhanced surveillance. The timely and coordinated execution of these post-efficacy tasks will bring the pandemic to an effective, and efficient, close.
\end{abstract}

T he severe acute respiratory syndrome coronavirus 2 (SARS-CoV-2) pandemic is a powerful reminder of the ability of infectious diseases to sicken, kill and disrupt, even in the most technologically advanced societies The rapid announcement of the outbreak and early publication of the viral sequence enabled work on a vaccine solution to proceed within weeks of China's initial notification of the World Health Organization (WHO) on 31 December 2019, of the outbreak ${ }^{1}$. Funding of grants for vaccine development from the Coalition for Epidemic Preparedness Innovations (CEPI) was announced in January 2020 (ref. ${ }^{2}$ ). Additional monies for vaccine development were also provided by national and multilateral research funders, with Operation Warp Speed (OWS) in the United States being the largest at $\$ 18$ billion $^{3}$ (Fig. 1).

Achieving the goal of development of a safe and efficacious vaccine had a 6-to 18-month timeline. The unprecedented effort has generated over 200 candidates in various stages of development, with over 50 candidate vaccines in human clinical trials and 18 in

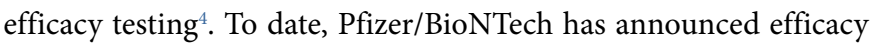
of $95 \%$; Gamaleya has announced efficacy of $92 \%$; Moderna has announced efficacy of $94.5 \%$; and AstraZeneca has announced efficacy of $70 \%$ 5,6. Sinopharm has now announced efficacy of $79 \%$, and several countries participating in the Sinovac (another Chinese company) efficacy trials have announced efficacies (for the same product) of $50 \%, 65 \%, 78 \%$ and $91 \%{ }^{7,8}$. Sinovac has yet to comment, and these data have not been published or peer reviewed. Pfizer/ BioNTech and Moderna are RNA vaccines expressing the coronavirus disease 2019 (COVID-19) spike glycoprotein, whereas vaccines from Gamaleya and AstraZeneca (partnered with Oxford University) express spike protein from adenovirus vector platforms. The vaccine developed by Gamaleya has a heterologous approach, with spike delivered in an adenovirus type 26 vector first, followed by a second dose containing spike in an adenovirus type 5 , and AstraZeneca uses a chimpanzee adenovirus-expressing spike. Sinopharm and Sinovac have whole inactivated virus vaccines with alum as an adjuvant. However, effective vaccination is one part of effective, comprehensive control of the pandemic. In addition, scientific, social and political questions-around dose, schedule, ethics, effectiveness, surveillance and vaccine hesitancy-remain to be resolved, and pandemic control will require that some, if not all, of these issues be solved intercurrently.

\section{Vaccine manufacturing}

Once shown to be safe and efficacious, a vaccine must be manufactured to the current good manufacturing practices (CGMP) international standard ${ }^{9,10}$. Current estimates of the level of effective population immunity for interruption of transmission is $\sim 60-70 \%$, but vaccination coverage required by a partially effective vaccine to interrupt transmission might be higher. With 8 billion people to vaccinate with a two-dose regimen, we might need 10-11 billion doses to interrupt transmission. CEPI estimates global vaccine manufacturing capacity at 2-4 billion doses annually, and that it will be 2023-2024 before enough vaccine can be manufactured. ${ }^{11}$ This capacity might be product specific, and there might be some limitations: whole-inactivated vaccines, for instance, must be manufactured in biosafety level 3-capable facilities. In addition to the administrative process of licensing and the subsequent technology transfer, the scale-up of vaccine manufacturing might be associated with unforeseen problems in the vector, purification or formulation.

Several of the companies with products in efficacy testing (phase $2 / 3$ or phase 3 ) have announced that they have started increasing production in anticipation of proof of efficacy and safety. Companies have sought partners for manufacturing to scale-up to the level of hundreds of millions of doses. AstraZeneca has partnered with Serum Institute of India and SK Bioscience (Republic of Korea) for its chimpanzee adenovirus-vectored SARS-CoV-2 vaccine. Sinovac (China) has partnered with Butantan (Brazil) and Bio Farma (Indonesia). Johnson \& Johnson has engaged Biological E (India). OWS has $\$ 1.6$ billion in arrangements with 'non-vaccine' manufacturers that include different contract manufacturers, including, for instance, the manufacture of medical glass for vaccine vials ${ }^{3}$.

Quality. Vaccine quality is often taken for granted, but both safety and efficacy can be affected by even minor lapses in quality. Country-specific regulatory requirements and a set of international standards developed by the International Council for Harmonisation of Technical Requirements for Pharmaceuticals for Human Use (ICH, https://www.ich.org/) provide the basis for ensuring quality in clinical research, while ICH Q series guidance ensures that vaccine quality meets global standards for chemistry, manufacturing and control. In addition, research laboratories supporting vaccine development must ensure alignment with Good

IInternational Vaccine Institute, Seoul, Republic of Korea. ${ }^{2}$ Cambridge Institute of Therapeutic Immunology and Infectious Disease, University of Cambridge School of Clinical Medicine, Cambridge, UK. ${ }^{3}$ University of Antananarivo, Antananarivo, Madagascar. ${ }^{4}$ International Centre for Diarrheal Diseases Research, Dhaka, Dhaka, Bangladesh. ${ }^{5}$ UCLA Fielding School of Public Health, Los Angeles, CA, USA. 凶e-mail: jerome.kim@ivi.int 


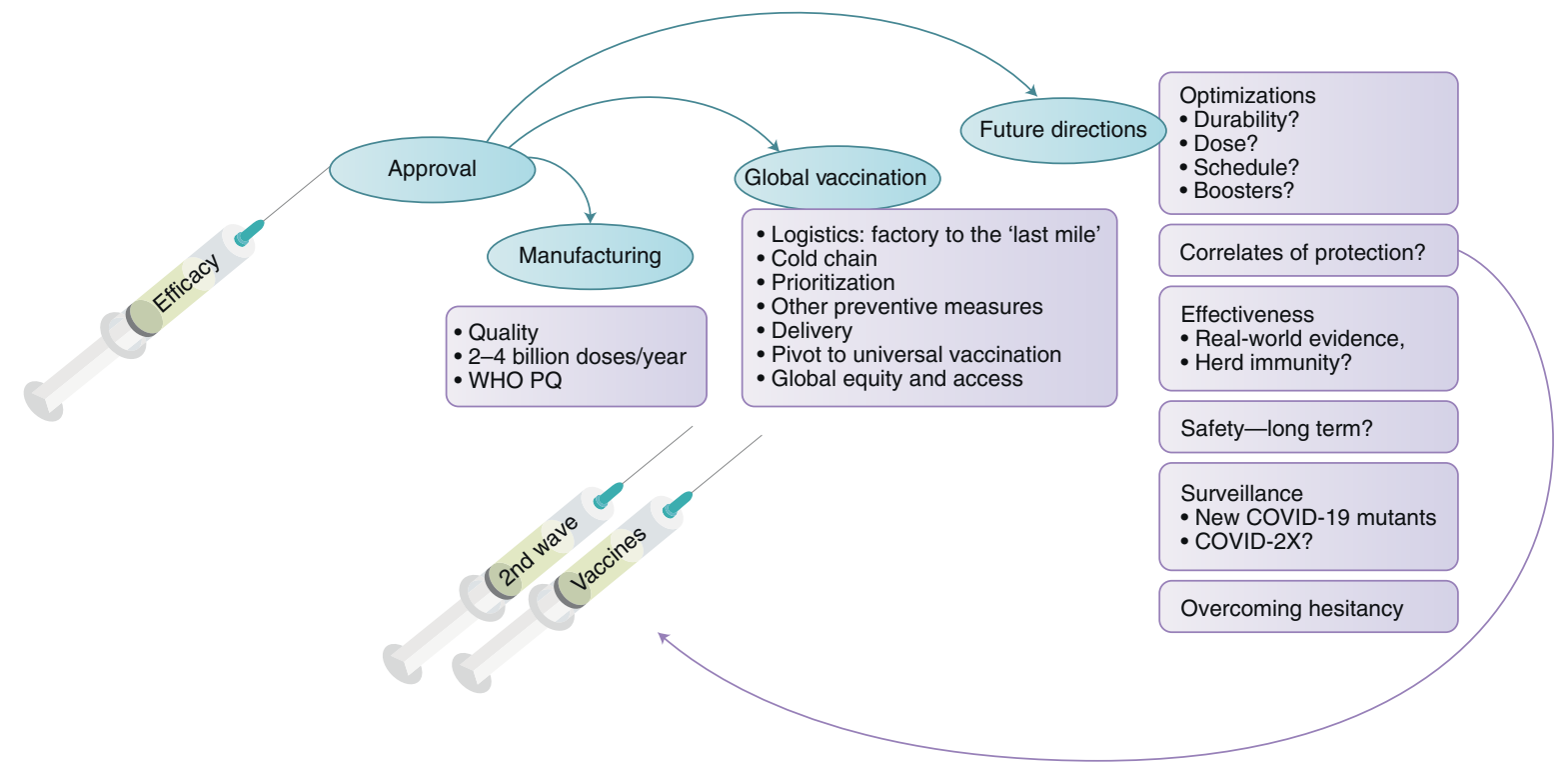

Fig. 1 | The next steps for a COVID-19 vaccine. After a COVID-19 vaccine has demonstrated efficacy in a clinical trial, the vaccine must be approved and manufacturing scaled up, according to an international standard known as CGMP. The transportation of the vaccine must respect the cold-chain requirements. COVID-19 vaccines should be allocated with respect to equity and access for LMICs. Several scientific questions around optimization of the vaccine(s) dose and schedule, boosting and correlates of protection must be answered, particularly if testing of the 'second wave' of vaccines cannot be accomplished with a placebo group (owing to licensure or approval of first-wave vaccines). Collection of effectiveness data and understanding indirect protective effects of vaccination will allow countries to make rational plans for maintaining herd protection. Surveillance for COVID-19 mutations and the sensitivity of those mutations to vaccine-induced immune responses will be necessary, as will continued vigilance for the emergence of new zoonotic coronavirus infections. Finally, for effective control of COVID-19 transmission, countries will need to address issues around vaccine hesitancy; increasing uptake of vaccines will be a priority before control over COVID-19 can be gained.

Laboratory Practice (for example, 21 CFR 58, OECD GLP), and, for human bioanalytical analysis, Good Clinical Laboratory Practice (GCLP) guidance is used. Collectively, these standards are referred to as GxPs (that is, Good Clinical Practice, Good Manufacturing Practice and Good Laboratory Practice) and form a common basis for quality and regulatory compliance. The effect of lapses in manufacturing quality has already been reported: differences between lots of the Sinovac vaccine ${ }^{12}$, the half-dose/full-dose regimen used by AstraZeneca ${ }^{6}$ and the lower response to the Sanofi/GSK vaccine in the elderly ${ }^{13}$. International standards for quality underpin the scientific validity of clinical trial data, the safety and immunogenicity of vaccine products and approval by regulatory agencies. Execution of clinical trials according to GCP is an international requirement for the sponsor, usually the vaccine manufacturer or, rarely, the funder of the research, and failure to do so might have legal, ethical, scientific and regulatory implications. Ensuring that vaccines are manufactured to international quality standards, for COVID-19 vaccines as well as other vaccines, provides assurance that the vaccines being deployed globally will have consistent safety and efficacy and will be critical to overcome vaccine hesitancy.

\section{Regulatory pathways}

Vaccines are approved in the country of manufacture by a national regulatory authority (NRA) - an organization like the US Food and Drug Administration (FDA) or the European Medicines Agency. NRAs are highly technical competencies, and not all NRAs meet the WHO/Global Fund to Fight AIDS, Tuberculosis and Malaria requirements to be a 'stringent' regulatory authority. ${ }^{14}$ The regulatory authorities in the United States, Europe, the United Kingdom and Japan are stringent, but those in India, South Korea, Brazil and Indonesia are deemed functional but not stringent. A vaccine approved by stringent or functional NRAs can be submitted for WHO pre-qualification (PQ) approval. The WHO does a second review of clinical, laboratory and manufacturing data, sometimes conducts inspections and, if satisfied, will issue a PQ approval. PQ approval allows United Nations agencies, such as the United Nations Children's Fund (UNICEF) or the Pan American Health Organization, to purchase the vaccines for global health use by organizations like Gavi, the Vaccine Alliance. WHO PQ is required for SARS-CoV-2 vaccines intended for COVAX, the vaccines pillar of the Access to COVID-19 Tools (ACT) Accelerator, established by the WHO, the European Commission and the French government. COVAX is jointly led by the WHO, Gavi and CEPI, and 189 countries have expressed interest in membership ${ }^{15}$. After PQ is granted, hundreds of millions of doses of vaccines from Indian, Indonesian, Brazilian and South Korean manufacturers can be bought by UNICEF, which is the largest purchaser of vaccines for Gavi, the Vaccine Alliance ${ }^{16}$. With the issuance of emergency use approvals and marketing authorizations, it is not clear whether countries whose NRAs lack the technical capacity to review dossiers will seek relief from COVID-19 by directly licensing vaccines without WHO review and purchase those vaccines directly from manufacturers through bilateral deals. The United Arab Emirates, for instance, has approved the emergency use of the Sinopharm whole-inactivated vaccine for healthcare workers in the absence of evidence of efficacy or safety but with an emergency use approval from the National Medical Products Agency of China.

\section{Vaccination access and equity}

Vaccines do not save lives; vaccination does. Gavi, the Vaccine Alliance, provides 57 countries with vaccines at low or no cost. For vaccines being moved from high-income country manufacturers to 
low-to-middle-income countries (LMICs), there are delays in vaccine implementation. Rotavirus vaccine was approved by the FDA in 2006 and recommended and approved by the WHO in 2009 , yet, in 2020 , only $60 \%$ of the world's children have received three doses of rotavirus vaccine. An 'access gap' for COVID-19 vaccines would have dramatic consequences; an analysis by the Duke Global Health Innovation Center suggests that more than 8 billion doses of vaccines under development from Western manufacturers have been pre-ordered by high-income countries ${ }^{17}$. The United States has pre-ordered 1.6 billion doses; the European Union has pre-ordered 1.5 billion doses; the United Kingdom has pre-ordered 400 million doses; and Japan has pre-ordered 300 million doses. One modeling study provides data that, if high-income countries exclusively acquire the first 2 billion doses without regard for vaccine equity, the number of COVID-19 deaths could double ${ }^{18}$.

COVAX is the vaccines pillar of the ACT and is led by the WHO; Gavi, the Vaccine Alliance; and CEPI ${ }^{19}$. COVAX intends to purchase 2 billion doses of WHO PQ-approved vaccine by the end of 2021 . The doses that COVAX intends to purchase should cover roughly $20 \%$ of each participating country's population, enough to vaccinate the elderly and healthcare workers, for instance. If the vaccine remains in short supply, COVAX will prioritize by country. The European Commission has provided $€ 400$ million for COVAX but will not use COVAX to purchase vaccine. Over 90 countries should qualify for free, or very low-cost, COVID-19 vaccines. The timeline for distribution of vaccines to LMICs is unknown at present but, the initial installment may be available as early as the first quarter of 2021, Each country should receive 3\% of anticipated need $^{20,21}$. It is not known how COVAX vaccine supply will be coordinated, because COVAX orders have to be considered in relation to expected vaccine deliveries from pre-ordered vaccines. A recent report from Reuters suggested that COVAX is at high risk of failure, and this puts LMICs at considerable jeopardy, although this was followed by an announcement by the WHO that, in principle, it had secured 2 billion doses of COVID-19 vaccines ${ }^{22,23}$.

At this point, AstraZeneca has committed to supplying vaccines to COVAX, and Pfizer and Moderna have not yet made announcements; ${ }^{21}$ some vaccine manufacturers are linked to COVAX through their receipt of CEPI funding, which requires a Global Access Agreement. President Xi Jinping of China also made verbal commitments during his comments to the WHO that might be relevant for Chinese companies. Because WHO PQ is required for distribution by COVAX, vaccines that might be approved by the European Medicines Agency or other stringent NRAs might be in the COVAX mechanism more quickly than vaccines developed by Russian or Chinese manufacturers; however, there could be other logistical problems, such as the requirement of a cold chain that might prevent LMICs from using certain types of vaccines available through COVAX. Among Western companies, this leaves the vaccine developed by AstraZeneca as the sole candidate that has reported efficacy and has features amenable to widespread use. Because of these logistical challenges, several LMICs have also placed orders for COVID-19 vaccines with Russian and Chinese manufacturers; China has offered \$1 billion in loans to Latin America; and President Xi has pledged $\$ 2$ billion to assist countries in the fight against COVID-19 (ref. ${ }^{24}$ ).

Beyond access to the vaccine, there are questions that are common to vaccines for global health ${ }^{10}$. The purchase, supply management, transport through the cold chain, in-country distribution and vaccine administration are accomplished daily for childhood vaccines with roughly $80 \%$ coverage worldwide, which is a testament to the success of national vaccination programs, complemented by the efforts of Gavi and other public and private entities ${ }^{25,26}$. Several factors complicate the use of COVID-19 vaccines: programmatic scale, in particular the need for vaccination of all age groups rather than simply children; logistics-a global supply chain that will be complex and might result in bottlenecks and delays; the potential use of multiple different vaccines and a lack of evidence on substitution of one for the other; and unique and demanding cold-chain requirements, especially for messenger RNA vaccines, which require $-20^{\circ} \mathrm{C}$ storage for the Moderna candidate and $-70^{\circ} \mathrm{C}$ storage for the Pfizer candidate (although higher temperatures are being explored), and which is an especially challenging issue for use in LMICs.

\section{Scientific questions}

Optimization of dose, schedule and boosters. The speed of vaccine development for SARS-CoV-2 has been unprecedented. However, the time that companies and investigators might have to explore dose optimization and schedule has been reduced by the urgency of development. Fractional dosing has already been used to extend the supply of yellow fever vaccine ${ }^{27,28}$, and, if lower doses of COVID-19 vaccines prove efficacious and safe, then, particularly when supplies are limited, fractional doses might allow more people to be vaccinated. AstraZeneca reported that a regimen of half dose followed at least 1 month later by a full dose of chimpanzee adenovirus-based COVID-19 vaccine resulted in higher efficacy than two full doses given at least 1 month apart ${ }^{6}$. Sinovac found that, for their whole-inactivated virus (WIV) vaccine, extending the COVID-19 vaccine dosing interval from 2 to 4 weeks might have improved neutralizing antibody titers ${ }^{12}$. Although closer spacing of doses might be advantageous during an outbreak, optimizing the schedule for magnitude and durability of induced responses might potentially make future COVID-19 vaccine campaigns more practical because they allow national programs time to plan and execute more effectively ${ }^{29,30}$.

We do not know yet whether protective immune responses are durable; perhaps identification of correlates of immune protection will determine what level of an immune biomarker is necessary for protection. Although the preponderance of evidence suggests that adaptive immune responses elicited by SARS-CoV-2 infection are present and might protect against re-infection ${ }^{31}$, experience with seasonal coronaviruses ${ }^{32}$ and present experience with SARS-CoV-2 suggest that immunity to natural infection might wane over time, and reinfection has been reported ${ }^{33,34}$. Additional booster doses might be necessary to extend the duration of protection; we do not know whether primary series and booster doses can or should be different. It is also unclear whether previously infected persons would benefit from vaccination, although, at this point, vaccination against SARS-CoV-2 should occur irrespective of infection status.

Correlate of protection. A correlate of protection is a vaccineinduced biomarker whose presence is associated with a lower risk of infection, and not all vaccines have correlates of protection ${ }^{35-37}$. For example, a hemagglutinin inhibition antibody titer of 1:40 is used to license influenza vaccines that vary from year to year ${ }^{38}$, whereas anti-polyribosylribitol phosphate antibody levels of $0.15 \mu \mathrm{g} \mathrm{ml}^{-1}$ or greater are required for licensing of Hemophilus influenza type b conjugate vaccines $^{39}$. An important secondary objective of phase 3 randomized trials will be testing of immune serum from volunteers who were vaccinated and became infected compared to a matched set of vaccinated volunteers who did not become infected ${ }^{36,40}$. Ironically, vaccines with high efficacy (such as human papillomavirus vaccines or the Pfizer and Moderna COVID-19 vaccines with 94-95\% efficacy) are sometimes problematic because there are too few 'vaccinated infected' volunteers to power a correlates analysis. If one is found, the correlate might then be used as a proxy for efficacy of the vaccine, providing a biomarker for dose, schedule and booster optimization. This immune correlate could be used, in lieu of clinical endpoints, to simplify and accelerate future vaccine development, reducing trial size and the cost of efficacy trials. This may be an important issue for the testing of second- and third-wave 
vaccines for COVID-19 (i.e., the vaccines whose interim efficacy results are due in mid-to late 2021). This presumes that the mechanism of protection is common; it might not apply, for instance, if the vaccine for which a correlate was determined was injectable, and the comparator is given orally.

Safety. There are certain safety-related events that, due to rarity or pathogenesis, might be detected only during longer-term surveillance for adverse events after immunization ${ }^{41,42}$. The FDA guidance for Emergency Use Authorization suggests a median duration of follow-up of phase 3 vaccine trial volunteers of 2 months ${ }^{43}$. Most events are expected to fall within that window after vaccination. For example, intussusception after rotavirus vaccine administration is a rare but well-studied event, occurring at a frequency of $1 / 20,000$ $1 / 100,000$ vaccinees, and a statistical association between intussusception and rotavirus vaccine administration was seen in some ${ }^{44}$ but not other ${ }^{45}$ meta-analyses. In high-income countries, it is rarely fatal, and, in LMICs, the benefit of vaccination in preventing illness and death in vaccinated infants is significant ${ }^{46}$. Previous pandemic prevention campaigns, such as the swine flu pandemics of 1976 or 2009, were followed by reports of very rare but serious adverse events, Guillain-Barre syndrome and narcolepsy ${ }^{47}$. Robust systems for the follow-up of safety, both during emergency use and after licensure, will be critical to uncovering any potential rare events.

Because pregnant women and children have not been included in clinical trials of COVID-19 vaccines, these studies must eventually be completed, both for efficacy and, importantly, for safety. RNA-based vaccines are a new platform, so there are no existing data on safety in children or in pregnancy. Adenovirus vaccines have been approved for Ebola (Ad26- and Ad5-based), but significant data on safety during pregnancy are not available ${ }^{48,49}$ Although WIV vaccines have been used for multiple diseases and are generally safe, testing of COVID-19 WIV in these populations is also necessary.

With SARS-CoV-2, two additional questions arose with respect to safety have arisen, due to the significant sequence homology between SARS-CoV-2 and SARS-CoV (the original SARS virus): the potential for antibody-dependent enhancement (ADE) and the potential for vaccine-associated enhanced respiratory disease $(\text { VAERD })^{41,50}$. The first is an in vitro phenomenon of enhanced viral entry or replication in the presence of vaccine-induced antibody. This might result in enhanced infection or increase in 'severe' infections in vaccinated individuals who are infected by the homologous coronavirus. Notably, this has not been described in current reports of SARS-CoV-2 vaccine phase 3 trials. Similarly, SARS-CoV-2 vaccines in animal models (mice, hamsters, ferrets and non-human primates) have protected against challenge, and VAERD has not been reported. With both ADE and VAERD, the effect of waning antibody titers after vaccination (or after infection) and potential safety signals is unknown, However, there is a cautionary tale in the deployment of a dengue vaccine where more severe dengue virus disease was seen in a subset of recipients as protective, vaccine-associated antibody decreased. ${ }^{51}$ A final potential concern comes from multi-system inflammatory syndrome in children (MIS-C) who are infected with COVID-19; some have warned that the occurrence of MIS-C should be carefully monitored after COVID-19 vaccination becomes widespread ${ }^{52}$.

Both ADE and VAERD, however, remain theoretical concerns in human volunteers who have received various COVID-19 vaccines and who become infected during the course of a study. Consensus recommendations regarding the importance of follow-up for monitoring of potential disease enhancement have been published ${ }^{41}$.

Epidemiology and burden of COVID-19 in LMICs. A key question about the epidemiology of COVID-19 has important implications for LMICs. Numbers of infections and COVID-19-related deaths appear to be much lower in Africa than in other parts of the world. Or, as we learned during the early days of HIV, are we not seeing cases because of lack of testing and case and death ascertainment? Some data would suggest that this is the case. Kenya has reported 93,000 cases of COVID-19; however, a nationwide blood bank survey estimated that $5 \%$ of Kenyans had been infected, implying a number closer to 2.5 million $^{53}$. Similarly, the absence of death registry data creates challenges in determining number of infections. The South African Medical Research Council noted that weekly excess deaths are trending 60\% higher than in 2019, suggesting that current COVID-attributable deaths might be greatly undercounted, and some estimates put the number of infections and deaths even higher ${ }^{54,55}$.

Surveillance of SARS-CoV-2 evolution and emerging coronaviruses. We do not know whether a SARS-CoV-2 vaccine, based on strains isolated in 2019 and 2020, will protect against SARS-CoV-2 strains circulating in 2021 or 2022 . Sequence variation in SARS-CoV-2 strains appears to be eight- to tenfold lower than influenza A and many hundred-fold lower than HIV type 1 (refs. ${ }^{56,57}$ ). This might mean that today's vaccines might protect against SARS-CoV-2 strains in the future, but we do not have sufficient evidence to say that either new introductions or genetic drift might produce a variant virus requiring a different vaccine strain. As with the D614G variant that swept through Europe in the spring of 2020, detection, isolation and sequencing of the strain allowed groups to test serum from vaccinated animals and humans against this mutant in neutralization assays. Analysis of breakthrough infections in vaccine trials, known as 'sieve' analysis, might also shed light on variants showing neutralization resistance. Neutralization resistance, particularly if neutralizing antibody is shown to be a correlate of protection, might require development of additional COVID-19 vaccines.

One of the lessons of this coronavirus outbreak should be that we must monitor zoonotic transmissions in this (and other) potential future pathogens. A US government-sponsored project, called Predict, was created in 2009 in response to the swine flu pandemic to identify potential zoonotic pathogens, but it was shut down in 2019. Would a similar project provide advanced warning of future pandemic threats? A global surveillance program could be used to monitor changes in circulating SARS-CoV-2 variants that might proactively inform the future development of new vaccines.

Efficacy, effectiveness and herd immunity. All of the current phase 3 trials are designed as individually randomized, placebo-controlled clinical trials (RCTs). These trials will help ensure that necessary data are generated as quickly and efficiently as possible while maintaining high ethical and scientific standards. Randomizing individuals rather than groups or clusters of individuals helps minimize sample size requirements and enhance practical elements of trial execution-logistics, expense and subject accrual times. This type of randomization also serves to isolate estimates of vaccine protection from herd protective effects and confines estimates of efficacy to direct vaccine protection of an individual, not a population ${ }^{58}$. Moreover, the current phase 3 trials are powered to detect protection against symptomatic infections. However, protection against severe COVID-19 disease and mortality is a key goal of implementing a COVID-19 vaccine in practice, and knowledge of vaccine protection against overall COVID-19 disease might not permit prediction of protection against severe disease. In addition, study populations often focus on young, healthy adults. Furthermore, in the studies themselves, close attention is paid to timely attendance of study visits, cold-chain requirements and study product administration. These variables might be difficult to control as vaccination of the general population is implemented. RCT designs might therefore overestimate the level of vaccine protection compared to 
Table 1 | Metrics for previously approved vaccines

\begin{tabular}{|c|c|c|c|c|c|}
\hline Infection & Basic reproduction number $\left(R_{0}\right)$ & Herd immunity threshold (\%) & Vaccine efficacy (\%) & Effectiveness (\%) & References \\
\hline Diphtheria & $6-7$ & 85 & 97 & $>95$ & 74,75 \\
\hline Measles & $12-18$ & $55-94$ & 94 & $90-95$ & 75 \\
\hline Pertussis & $12-17$ & $92-94$ & $70-90$ & $75-85$ & 75 \\
\hline Polio & $12-15$ & $50-93$ & $80-90$ & $>90$ & 75 \\
\hline Rubella & $6-7$ & $83-85$ & $94-95$ & $>95$ & 75 \\
\hline Ebola & $1.5-2.5$ & $33-60$ & $95-100$ & 70 & 71 \\
\hline Varicella & $8-10$ & $87-90$ & $90-98$ & $>95$ & 75 \\
\hline Spanish flu 1918 & $2-3$ & $50-67$ & NA & NA & 76 \\
\hline Cholera & $1-2$ & 50 & $42-66$ & 86 & $77-79$ \\
\hline SARS-CoV-2 & $2.5-5.8$ & $60-83$ & $60-95$ & $?$ & 76,80 \\
\hline
\end{tabular}

real-world settings, with a wider spectrum of vaccinees and less meticulous control over logistical and vaccine administration. In the 'real world', a person might arrive 3 weeks late for the second vaccine dose, or the vaccine might have been in a refrigerator or freezer that had been unmonitored and had a significant excursion of temperature; these deviations would be problematic in a clinical trial.

Conversely, RCTs might underestimate the protective effect of vaccines at the population level. This would occur if the COVID19 vaccine, in addition to conferring direct protection to individuals, reduces transmission of COVID-19 between individuals, providing protection to unvaccinated individuals and enhanced protection of vaccinated individuals in contact with vaccinated individuals. Vaccine-induced herd protection, which might be crucial to the public health value of a vaccine, will be missed when trials are individually randomized and analyses fail to take account of the geographical distribution of individuals in the population ${ }^{58}$. Table 1 provides a summary of estimates of the basic reproduction number, herd immunity, efficacy and effectiveness for several vaccine-preventable diseases. The definition of effectiveness might vary by study.

For these reasons, even when COVID-19 vaccines have achieved licensure via current phase 3 trials, there will be substantial uncertainties about how useful the vaccines will be in practice, and studies done after licensure, addressing vaccine effectiveness, including the level of protection of both vaccinated and non-vaccinated individuals in entire targeted populations, will be needed ${ }^{58,59}$. Several designs can be considered. A common post-licensure approach is simply to monitor the secular trend of cases against the concomitant secular trend of vaccine coverage ${ }^{60,61}$. This approach suffers from several potential, not easily correctible, biases, not least that it assumes that the underlying trend of cases, independent of vaccination, can be predicted, which will be difficult for COVID-19. Both a cohort design, in which COVID-19 vaccinees and non-vaccinees would be followed in time for the comparative occurrence of COVID-19, and a case-control design, in which COVID-19 cases and non-COVID-19 controls would be compared for the antecedent receipt of COVID19 vaccine, are commonly used to evaluate vaccine protection after licensure $^{62}$. These designs are less susceptible to biases related to the variation of disease incidence over time for natural or manmade (non-vaccine) reasons but are susceptible to biased estimation of vaccine protection owing to differences in who receives or does not receive vaccine, ascertainment of vaccination and interventions received by vaccinated versus non-vaccinated people, as well as potentially unequal surveillance for detection and diagnosis of the target disease. Fortunately, such biases can be mitigated, or at least measured, at various stages of the study ${ }^{61-64}$. Although these designs provide vaccine protection estimates under realistic public health conditions, conventional analyses of these studies generally do not permit assessment of population-level vaccine protection, including vaccine herd protection. However, recent methodological advances in the design and analysis of these studies, employing GIS mapping to enable analyses within defined virtual geographic clusters, offer the opportunity to estimate these population-level effects ${ }^{65}$.

Another potential option for measuring population-level vaccine protection against COVID-19, when a licensed vaccine is available, is the cluster-randomized effectiveness trial (CRTs), in which some clusters of individuals are randomized to receive the study vaccine in a way that simulates public health practice, whereas other clusters of individuals are randomized to not receive the vaccine, such as that from a CRT of typhoid polysaccharide vaccine in Kolkata ${ }^{66}$. CRTs usually provide the best protection against bias in measuring population-level vaccine protective effects ${ }^{59,64,67,68}$. However, CRTs can be done only in settings where it is scientifically and ethically acceptable to intentionally not give the vaccine under study to control clusters, which might pose a considerable dilemma for studies of COVID-19 vaccines during a pandemic. The use of placebos in future clinical trials will undoubtedly be complicated given the availability of other vaccines after licensure ${ }^{69}$.

CRTs can also be executed using a stepped-wedge approach, which was first used in Gambia for the introduction of hepatitis B vaccine $^{70}$, in which clusters of individuals are randomly phased in to receive the vaccine under study over time, or parallel designs, in which clusters of individuals are simultaneously randomized to receive the vaccine or a comparator (another vaccine or no vaccine). In addition, experience with the Ebola vaccine demonstrates the potential for CRTs to be used in the evaluation of vaccines in outbreak settings ${ }^{71}$.

\section{The end of the beginning}

It will be important for vaccination, initially, to be seen as one part of a comprehensive package of COVID-19 prevention, which will include masks, distancing, hygiene and preparedness of healthcare facilities for an unknown period. Will misinformation, vaccine hesitancy, politics, deception and division undermine efforts to vaccinate communities and lower the burden of COVID-19? Global acceptability of vaccines varies from a low of $55 \%$ in Russia to a high of $90 \%$ in China $^{72}$. The WHO has issued a report highlighting the importance of up-to-date scientific information as the context in which an enabling environment, positive social influences and 
motivation will be critical to achieve high vaccine uptake, but this critical and complex subject is best pursued separately ${ }^{73}$. For vaccination campaigns to be successful, governments must anticipate these issues and build trust and consensus around the safety, efficacy and necessity of vaccination.

In summary, vaccines are an important tool, but they must be wielded effectively and used in conjunction with other evidence-based public health measures to be decisive. A comprehensive program of prevention, continued work on vaccine optimization, new vaccines, correlates, long-term safety and continued surveillance will be needed simultaneously with steady implementation of vaccination. Understanding effectiveness will require systematic implementation of post-licensure studies to understand the key parameters around herd immunity and polices derived from that knowledge. Safe and effective COVID-19 vaccines were developed in 11 months. In the next year, effective vaccination and prevention efforts could prove decisive, and, although the work ahead is considerable, perhaps the 'end of the beginning' is at hand.

Received: 2 November 2020; Accepted: 6 January 2021; Published online: 19 January 2021

\section{References}

1. World Health Organization. Novel Coronavirus (2019-nCoV), Situation Report - 1. https://www.who.int/docs/default-source/coronaviruse/ situation-reports/20200121-sitrep-1-2019-ncov.pdf (2020).

2. Coalition for Epidemic Preparedness Innovations. CEPI to fund three programmes to develop vaccines against the novel coronavirus, nCoV-2019. https://cepi.net/news_cepi/cepi-to-fund-three-programmes-to-developvaccines-against-the-novel-coronavirus-ncov-2019/ (2020).

3. Baker, S. \& Coons, C. Inside Operation Warp Speed's $\$ 18$ billion sprint for a vaccine. Bloomberg Business Week https://www.bloomberg.com/news/features/ 2020-10-29/inside-operation-warp-speed-s-18-billion-sprint-for-a-vaccine (2020).

4. Parker, E. P. K., Shrotri, M. \& Kampmann, B. Keeping track of the SARS-CoV-2 vaccine pipeline. Nat. Rev. Immunol. 20, 650 (2020).

5. Polack, F. P. et al. Safety and efficacy of the BNT162b2 mRNA Covid-19 vaccine. N. Engl. J. Med. 383, 2603-2615 (2020).

6. Voysey, M. et al. Safety and efficacy of the ChAdOx1 nCoV-19 vaccine (AZD1222) against SARS-CoV-2: an interim analysis of four randomised controlled trials in Brazil, South Africa, and the UK. Lancet 397, 99-111 (2020).

7. Reuters. Sinopharm's COVID-19 vaccine $79 \%$ effective, seeks approval in China. https://www.reuters.com/article/us-health-coronavirus-china-vaccine/ sinopharms-covid-19-vaccine-79-effective-seeks-approval-in-chinaidUSKBN2940C8 (2020).

8. BBC. Sinovac: Brazil results show Chinese vaccine $50.4 \%$ effective. https:// www.bbc.com/news/world-latin-america-55642648 (2020).

9. Plotkin, S., Robinson, J. M., Cunningham, G., Iqbal, R. \& Larsen, S. The complexity and cost of vaccine manufacturing-an overview. Vaccine 35, 4064-4071 (2017).

10. Smith, J., Lipsitch, M. \& Almond, J. W. Vaccine production, distribution, access, and uptake. Lancet 378, 428-438 (2011).

11. Coalition for Epidemic Preparedness Innovations. CEPI survey assesses potential COVID-19 vaccine manufacturing capacity. https://cepi.net/news cepi/cepi-survey-assesses-potential-covid-19-vaccine-manufacturing-capacity/ (2020).

12. Zhang, Y. et al. Safety, tolerability, and immunogenicity of an inactivated SARS-CoV-2 vaccine in healthy adults aged 18-59 years: a randomised, double-blind, placebo-controlled, phase 1/2 clinical trial. Lancet Infect. Dis. https://doi.org/10.1016/S1473-3099(20)30843-4 (2020).

13. Sanofi and GSK announce a delay in their adjuvanted recombinant protein-based COVID-19 vaccine programme to improve immune response in the elderly. https://www.gsk.com/en-gb/media/press-releases/sanofi-andgsk-announce-a-delay-in-their-adjuvanted-recombinant-protein-based-covid19-vaccine-programme-to-improve-immune-response-in-the-elderly/ (2020).

14. World Health Organization. List of stringent regulatory authorities (SRAs). https://www.who.int/medicines/regulation/sras/en/ (2020).

15. Usher, A. D. COVID-19 vaccines for all? Lancet 395, 1822-1823 (2020).

16. Jadhav, S., Gautam, M. \& Gairola, S. Role of vaccine manufacturers in developing countries towards global healthcare by providing quality vaccines at affordable prices. Clin. Microbiol, Infect. 20, 37-44 (2014).

17. Duke Global Health Innovation Center. Mapping COVID-19 vaccine prepurchases across the globe. https://launchandscalefaster.org/COVID-19 (2020).
18. Chinazzi, M. et al. Estimating the effect of cooperative versus uncooperative strategies of COVID-19 vaccine allocation: a modeling study. https://www. networkscienceinstitute.org/publications/estimating-the-effect-ofcooperative-versus-uncooperative-strategies-of-covid-19-vaccine-allocationa-modeling-study (2020).

19. Gavi. The Gavi COVAX AMC explained. https://www.gavi.org/vaccineswork/ gavi-covax-amc-explained (2020).

20. Kupferschmidt, K. Despite obstacles, WHO unveils plan to distribute vaccine. Science 369, 1553 (2020)

21. Cuddy, A. Coronavirus vaccines: will any countries get left out? https://www. bbc.com/news/world-54961045 (2020).

22. Guarascio, F. Exclusive-WHO vaccine scheme risks failure, leaving poor countries with no COVID shots until 2024. https://www.nasdaq.com/ articles/exclusive-who-vaccine-scheme-risks-failure-leaving-poorcountries-no-covid-shots-until (2020).

23. Higgins-Dunn, N. COVAX global Covid vaccine program secures nearly 2 billion doses as UNICEF prepares for distribution. https://www.cnbc.com/ 2020/12/18/covax-global-covid-vaccine-program-secures-nearly-2-billiondoses-for-unicef-distribution.html (2020).

24. Freyman, E. \& Stebbing, J. China is winning the vaccine race. Foreign Affairs https://www.foreignaffairs.com/articles/united-states/2020-11-05/chinawinning-vaccine-race (2020).

25. Greenwood, B. The contribution of vaccination to global health: past, present and future. Philos. Trans. R. Soc. Lond. B Biol. Sci. 369, 20130433 (2014).

26. Mantel, C. \& Cherian, T. New immunization strategies: adapting to global challenges. Bundesgesundheitsblatt Gesundheitsforschung Gesundheitsschutz 63, 25-31 (2020).

27. Roukens, A. H., Vossen, A. C., Bredenbeek, P. J., van Dissel, J. T. \& Visser, L. G. Intradermally administered yellow fever vaccine at reduced dose induces a protective immune response: a randomized controlled non-inferiority trial. PLoS ONE 3, e1993 (2008).

28. Wu, J. T., Peak, C. M., Leung, G. M. \& Lipsitch, M. Fractional dosing of yellow fever vaccine to extend supply: a modelling study. Lancet $\mathbf{3 8 8}$, 2904-2911 (2016).

29. Holl, K., Sauboin, C., Amodio, E., Bonanni, P. \& Gabutti, G. Coverage, efficacy or dosing interval: which factor predominantly influences the impact of routine childhood vaccination for the prevention of varicella? A model-based study for Italy. BMC Public Health 16, 1103 (2016).

30. Lofano, G., Mallett, C. P., Bertholet, S. \& O’Hagan, D. T. Technological approaches to streamline vaccination schedules, progressing towards single-dose vaccines. NPJ Vaccines 5, 88 (2020).

31. Wajnberg, A. et al. Robust neutralizing antibodies to SARS-CoV-2 infection persist for months. Science 370, 1227-1230 (2020).

32. Edridge, A. W. D. et al. Seasonal coronavirus protective immunity is short-lasting. Nat. Med. 26, 1691-1693 (2020).

33. Iwasaki, A. What reinfections mean for COVID-19. Lancet Infect. Dis. 21, 3-5 (2020).

34. de Vrieze, J. More people are getting COVID-19 twice, suggesting immunity wanes quickly in some. https://www.sciencemag.org/news/2020/11/morepeople-are-getting-covid-19-twice-suggesting-immunity-wanes-quickly-some (2020)

35. Addetia, A. et al. Neutralizing antibodies correlate with protection from SARS-CoV-2 in humans during a fishery vessel outbreak with high attack rate. J. Clin. Microbiol. 58, e12107-20 (2020).

36. Plotkin, S. A. Updates on immunologic correlates of vaccine-induced protection. Vaccine 38, 2250-2257 (2020).

37. Yu, J. et al. DNA vaccine protection against SARS-CoV-2 in rhesus macaques. Science 369, 806-811 (2020).

38. Cox, R. J. Correlates of protection to influenza virus, where do we go from here? Hum. Vaccin. Immunother. 9, 405-408 (2013).

39. Denoel, P. A. et al. Quality of the Haemophilus influenzae type b (Hib) antibody response induced by diphtheria-tetanus-acellular pertussis/Hib combination vaccines. Clin. Vaccin. Immunol. 14, 1362-1369 (2007).

40. Plotkin, S. A. \& Gilbert, P. B. Nomenclature for immune correlates of protection after vaccination. Clin. Infect. Dis. 54, 1615-1617 (2012).

41. Lambert, P. H. et al. Consensus summary report for CEPI/BC March 12-13, 2020 meeting: assessment of risk of disease enhancement with COVID-19 vaccines. Vaccine 38, 4783-4791 (2020).

42. Definition and Application of Terms for Vaccine Pharmacovigilance. Report of CIOMS/WHO Working Group on Vaccine Pharmacovigilance https://www. who.int/vaccine_safety/initiative/tools/CIOMS_report_WG_vaccine.pdf (2012).

43. Krause, P. R. \& Gruber, M. F. Emergency use authorization of Covid vaccinessafety and efficacy follow-up considerations. N. Engl. J. Med. 383, e106 (2020).

44. Koch, J., Harder, T., von Kries, R. \& Wichmann, O. Risk of intussusception after rotavirus vaccination. Dtsch. Arztebl. Int. 114, 255-262 (2017).

45. Lu, H.-L., Ding, Y., Goyal, H. \& Xu, H.-G. Association between rotavirus vaccination and risk of intussusception among neonates and infants: a systematic review and meta-analysis. JAMA Netw. Open 2, e1912458-e1912458 (2019). 
46. Clark, A. et al. Mortality reduction benefits and intussusception risks of rotavirus vaccination in 135 low-income and middle-income countries: a modelling analysis of current and alternative schedules. Lancet Glob. Health 7, e1541-e1552 (2019).

47. Trombetta, C. M., Gianchecchi, E. \& Montomoli, E. Influenza vaccines: evaluation of the safety profile. Hum. Vaccin. Immunother. 14, 657-670 (2018).

48. Jacob, S. T. et al. Ebola virus disease. Nat. Rev. Dis. Prim. 6, 13 (2020)

49. World Health Organization. Ebola vaccine candidates. https://www.who.int/ blueprint/priority-diseases/key-action/ebola-vaccine-candidates/en/ (2019).

50. Zellweger, R. M., Wartel, T. A., Marks, F., Song, M. \& Kim, J. H. Vaccination against SARS-CoV-2 and disease enhancement - knowns and unknowns. Expert Rev. Vaccines 19, 691-698 (2020).

51. Sridhar, S. et al. Effect of dengue serostatus on dengue vaccine safety and efficacy. N. Engl. J. Med. 379, 327-340 (2018).

52. Kamidani, S. \& Pickering, L. Surveillance, research needed to identify optimal treatments for MIS-C. AAP News 41, 12 (2020).

53. Uyoga, S. et al. Seroprevalence of anti-SARS-CoV-2 IgG antibodies in Kenyan blood donors. Science 371, eabe1916 (2020).

54. Yam, E. Why we don't know the real number of COVID-19 deaths in Africa. International Growth Centre. https://www.theigc.org/blog/why-we-dontknow-the-real-number-of-covid-19-deaths-in-africa/ (2020).

55. Sguazzin, A. SA study puts Covid deaths much higher than official toll. https://www.moneyweb.co.za/news/south-africa/sa-study-puts-coviddeaths-much-higher-than-official-toll/ (2020).

56. Dearlove, B. et al. A SARS-CoV-2 vaccine candidate would likely match all currently circulating variants. Proc. Natl Acad. Sci. USA 117, 23652-23662 (2020).

57. Korber, B. et al. Evolutionary and immunological implications of contemporary HIV-1 variation. Br. Med. Bull. 58, 19-42 (2001).

58. Clemens, J., Brenner, R., Rao, M., Tafari, N. \& Lowe, C. Evaluating new vaccines for developing countries. Efficacy or effectiveness? JAMA 275, 390-397 (1996).

59. Wilder-Smith, A. et al. The public health value of vaccines beyond efficacy: methods, measures and outcomes. BMC Med. 15, 138 (2017).

60. Sullivan, S. G. \& Cowling, B. J. 'Crude vaccine effectiveness' is a misleading term in test-negative studies of influenza vaccine effectiveness. Epidemiology 26, e60 (2015).

61. Ainslie, K. E. C., Haber, M. \& Orenstein, W. A. Challenges in estimating influenza vaccine effectiveness. Expert Rev. Vaccines 18, 615-628 (2019).

62. Verani, J. R. et al. Case-control vaccine effectiveness studies: preparation, design, and enrollment of cases and controls. Vaccine 35, 3295-3302 (2017).

63. Haber, M., Watelet, L. \& Halloran, M. E. On individual and population effectiveness of vaccination. Int. J. Epidemiol. 24, 1249-1260 (1995).

64. Lucas, M. E. et al. Effectiveness of mass oral cholera vaccination in Beira, Mozambique. N. Engl. J. Med. 352, 757-767 (2005).

65. Ali, M. \& Clemens, J. Assessing vaccine herd protection by killed whole-cell oral cholera vaccines using different study designs. Front. Public Health 7, 211 (2019).

66. Sur, D. et al. A cluster-randomized effectiveness trial of Vi typhoid vaccine in India. N. Engl. J. Med. 361, 335-344 (2009).

67. Hitchings, M. D. T., Lipsitch, M., Wang, R. \& Bellan, S. E. Competing effects of indirect protection and clustering on the power of cluster-randomized controlled vaccine trials. Am. J. Epidemiol. 187, 1763-1771 (2018).
68. Kahn, R., Rid, A., Smith, P. G., Eyal, N. \& Lipsitch, M. Choices in vaccine trial design in epidemics of emerging infections. PLoS Med. 15, e1002632 (2018).

69. World Health Organization. Expert consultation on the use of placebos in vaccine trials. https:/www.who.int/ethics/publications/9789241506250/en/ (2013).

70. Peto, T. J. et al. Efficacy and effectiveness of infant vaccination against chronic hepatitis B in the Gambia Hepatitis Intervention Study (1986-90) and in the nationwide immunisation program. BMC Infect. Dis. 14, 7 (2014).

71. Henao-Restrepo, A. M. et al. Efficacy and effectiveness of an rVSV-vectored vaccine in preventing Ebola virus disease: final results from the Guinea ring vaccination, open-label, cluster-randomised trial (Ebola Ça Suffit!). Lancet 389, 505-518 (2017).

72. Lazarus, J. V. et al. A global survey of potential acceptance of a COVID-19 vaccine. Nat. Med. https://doi.org/10.1038/s41591-020-1124-9 (2020).

73. Behavioural considerations for acceptance and uptake of COVID vaccines. http://amarhealth.com/Spot-Light/5575/Behavioural-considerations-foracceptance-and-uptake-of-COVID-vaccines (2020).

74. Bisgard, K. M. et al. Diphtheria toxoid vaccine effectiveness: a case-control study in Russia. J. Infect. Dis. 181, S184-S187 (2000).

75. Plotkin, S. A., Orenstein, W. A. \& Offit, P. A. E. Vaccines, 6th edn (Elsevier, 2013).

76. Petersen, E. et al. Comparing SARS-CoV-2 with SARS-CoV and influenza pandemics. Lancet Infect. Dis. 20, e238-e244 (2020).

77. $\mathrm{Bi}, \mathrm{Q}$. et al. Protection against cholera from killed whole-cell oral cholera vaccines: a systematic review and meta-analysis. Lancet Infect. Dis. 17, 1080-1088 (2017).

78. Longini, I. M. Jr. et al. Controlling endemic cholera with oral vaccines. PLoS Med. 4, e336-e336 (2007).

79. Mukandavire, Z. \& Morris, J. G. Modeling the epidemiology of cholera to prevent disease transmission in developing countries. Microbiol. Spectr. https://scite.ai/reports/10.1128/ microbiolspec.VE-0011-2014 (2015).

80. Gomes, M. G. M., et al. Individual variation in susceptibility or exposure to SARS-CoV-2 lowers the herd immunity threshold. Preprint at medRxiv https://doi.org/10.1101/2020.04.27.20081893 (2020).

\section{Competing interests}

J.H.K. is a consultant for SK Biosciences.

\section{Additional information}

\section{Correspondence should be addressed to J.H.K.}

Peer review information Hannah Stower was the primary editor on this article and managed its editorial process and peer review in collaboration with the rest of the editorial team.

Reprints and permissions information is available at www.nature.com/reprints.

Publisher's note Springer Nature remains neutral with regard to jurisdictional claims in published maps and institutional affiliations.

(c) Springer Nature America, Inc. 2021 